\title{
Epigenomic Hard Drive Imprinting: A Hidden Code Beyond the Biological Death of Cancer Patients
}

REVIEW

\author{
Pritish Nilendu, Nilesh Kumar Sharma \\ Cancer and Translational Research Lab, Dr. D. Y. Patil Biotechnology and Bioinformatics Institute, Dr. D. Y. Patil Vidyapeeth, Maharashtra, India
}

\begin{abstract}
Several genetic and epigenetic theories have been suggested to explain the intricacies of life and death. However, several questions remain unsettled regarding cellular death events, particularly of living tissue in the case of cancer patients, such as the fate and adaptation of cancer cells after biological death. It is possible that cancer cells can display the intent to communicate with the external environment after biological death by means of molecular, genetic, and epigenetic pathways. Whether these cancer cells contain special information in the form of coding that may help them survive beyond the biological death of cancer patients is unknown. To understand these queries in the cancer field, we hypothesize the epigenomic hard drive (EHD) as a cellular component to record and store global epigenetic events in cancerous and non-cancerous tissues of cancer patients. This mini-review presents the novel concept of EHD that is reinforced with the existing knowledge of genetic and epigenetic events in cancer. Further, we summarize the EHD understanding that may impart much potential and interest for basic and clinical scientists to unravel mechanisms of carcinogenesis, therapeutic markers, and differential drug responses.
\end{abstract}

(J Cancer Prev 2017;22:211-218)

Key Words: Chromatin, Death, Environment, Epigenomics, Neoplasms

\section{INTRODUCTION}

Since the discovery of proto-oncogenes, key cellular proteins behind cancer initiation, development and progression have been reported to control trillions of cellular units in the human body. ${ }^{1.2}$ Emerging evidence and theories converge to a common consensus that the composition of these proto-oncogene encoded players, as well as tumor suppressors, could be perturbed by several internal and external clues. ${ }^{1.5}$ Genetic, epigenetic, molecular, and signaling disturbances change the behavior, tendency, and nature of certain human cell populations into an aberrant state..$^{3.7}$ These small populations of cells are commonly described as cancerous or malignant cells. Interestingly, these cancerous cells do not prefer to move away from the cellular community; instead, they invade, weaken and deprive healthy tissues. Finally, cancer cells create pro-growth and survival tumor microenvironment (TME) to communicate with the internal and external milieu. ${ }^{1,2}$ This mini-review highlights the important aspects of the dynamic nature of epigenomic hard drive (EHD) of tumor tissue before and after the biological death of cancer patients in the perspectives of heterogeneous microenvironments. This mini-review also emphasizes the clinical and translational impact of carcinoma therapeutics.

\section{EPIGENOMIC HARD DRIVE IMPRINTING OF CANCER CELLS BEFORE AND AFTER BIOLOGICAL DEATH}

It is well accepted that cancer cells destroy neighboring healthy cells and can lead to the collapse and death of cancer patients. In this circumstance, it may be important to know whether these

Received November 3, 2017, Revised December 7, 2017, Accepted December 11, 2017

Correspondence to: Nilesh Kumar Sharma

Cancer and Translational Research Lab, Biotechnology and Bioinformatics Institute, Dr. D. Y. Patil Vidyapeeth, Pune-411033, Maharashtra, India Tel: +91-02067919463, Fax: +91-67919622, E-mail: nilesh.sharma@dpu.edu.in, ORCID: Nilesh Kumar Sharma, http://orcid.org/0000-0002-8774-3020

Copyright (C) 2017 Korean Society of Cancer Prevention

(c) This is an Open Access article distributed under the terms of the Creative Commons Attribution Non-Commercial License (http://creativecommons.org/licenses/by-nc/4.0) which permits unrestricted non-commercial use, distribution, and reproduction in any medium, provided the original work is properly cited. 
cancer cells are aware that destroying healthy tissues may lead to themselves being disposed to death. Hence, there is a need to propose the existence of some coded language in the form of EHD imprinting to reveal communication between cancer cells and their microenvironment. In a recent study, DNA hypomethylation was shown to contribute to the development of cancer through the deletion or loss of tumor suppressors. ${ }^{8}$ Thus, these cellular events may lead to the modification of the chemical structure of DNA and may contribute to EHD. Other curiosities arise that once the human body faces biological death, what are the possibilities that these cancerous cells will present resistance to cell death. The schematic model of EHD in cancer tissues before and after biological death is presented in Figure 1..$^{3-6}$

In every organism, genomic and epigenomic events are orchestrated by dedicated intracellular and extracellular components. In an outstanding paper, Erlich and Zielinski ${ }^{9}$ claimed that DNA can display the digital information capacity. This study indirectly raised the possibility of the existence of an epigenetic hard drive within the healthy and cancerous cells of cancer patients. Essentially, these data and hypotheses revisit the knowledge of DNA as the fundamental unit for stored information in cells. ${ }^{10}$ In this proposed view, we extend this existing knowledge into a perspective for the cellular existence of some epigenetic recorder molecules to act as chromatin remodeling factors. These epigenetic recorders may record every epigenetic event inside the heterogeneous cellular components of cancer patients. Clearly, these epigenetic events can be stated as likelihood of a gene to being turned on or off due to epigenetic processes, such as methylation, phosphorylation, and acetylation. Further, gene regulation by various genetic and epigenetic events raises an intriguing question about the frequency of chromatin alterations and the possibility of this information that may potentially be stored in cells. Further, there is interest in the frequency of modeling and remodeling (change in the shape of chromatin) via histone protein tail methylation, acetylation, phosphorylation and other forms of chemical alterations. All of these regulatory events occurring inside normal and cancerous cells may be stored in the form of coded data within chromatin structure. In this perspective paper, we suggest designating this epigenetic information stored and coded in the form of an EHD. Imprinting of this information (coding) is referred to as EHD imprinting. As cancer is a part of the body, every single event by these cells may be recorded by the recorder molecules in the form of an EHD. Deriving information about these events through EHD imprinting may serve as a convincing tool for cancer therapeutics. A schematic viewpoint is illustrated in Figure 2 and $3 .^{3-8}$

\section{IMPORTANCE OF EPIGENOMIC HARD DRIVE IMPRINTING}

There is a lack of knowledge about genetic, signaling and epigenetic events contributing to the survival of cancer cells after

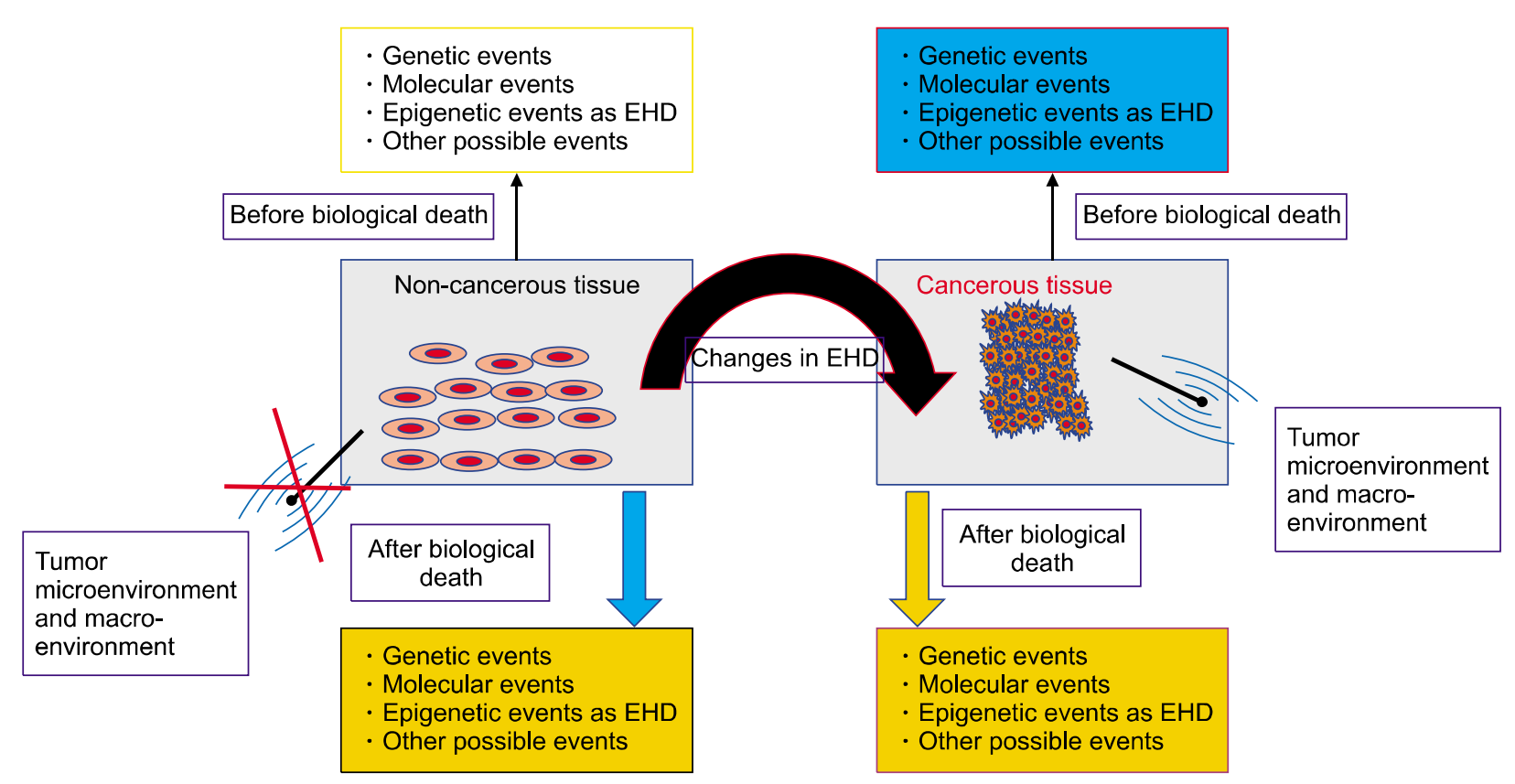

Figure 1. The schematic representation of epigenomic hard drive (EHD) in tumor and normal tissue. This model takes into account of tumor microenvironment and macroenvironment contribution in shaping of EHD in tumor tissue. 
A

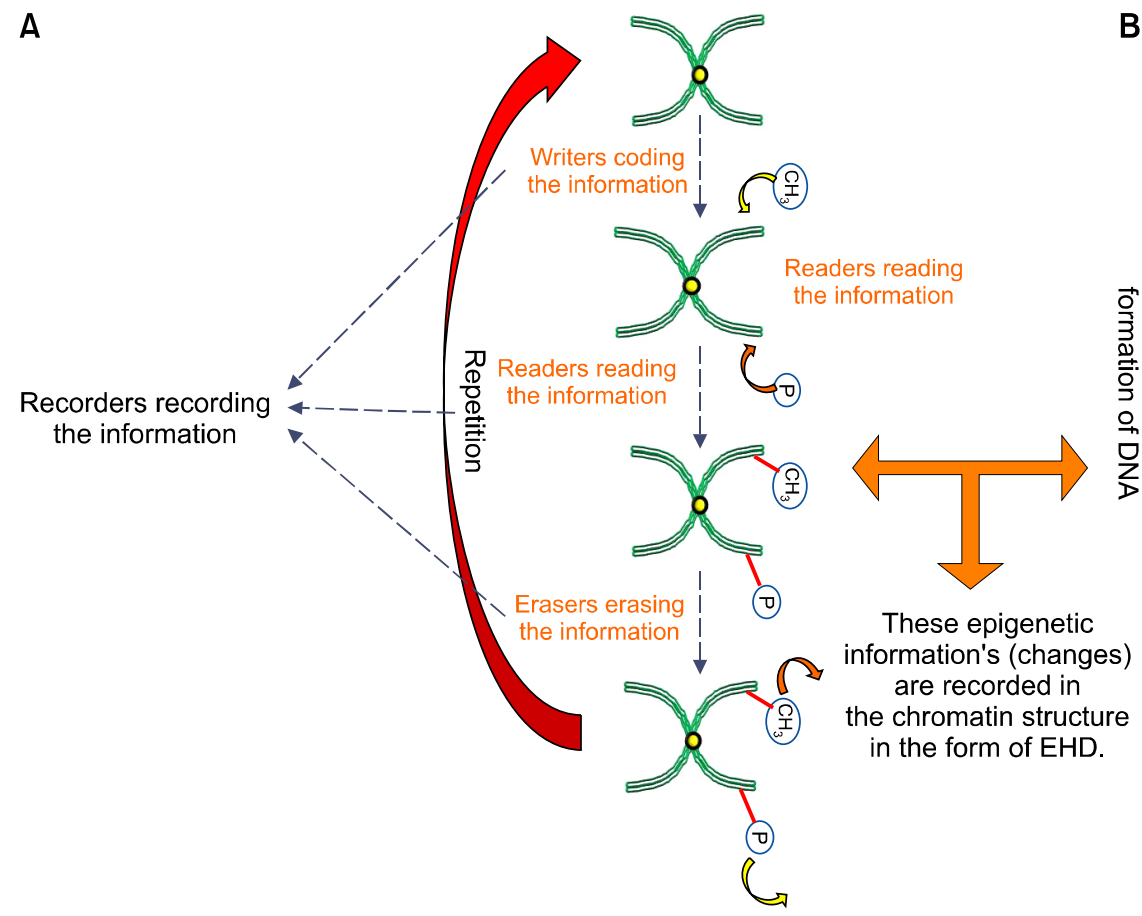

B Recorders recording

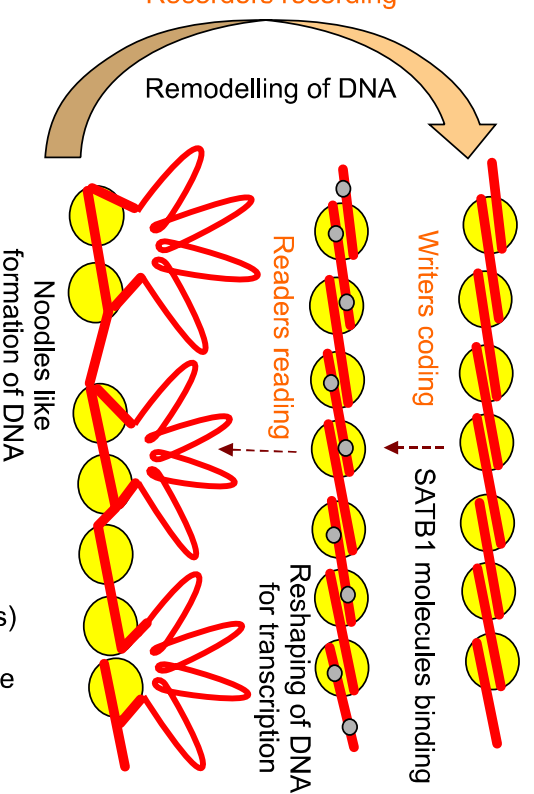

Figure 2. Epigenomic hard drive (EHD) during chromatin alterations. (A) Diagram describes that a methyl and a phosphate group approach, causing addition of these groups respectively to chromatin. As this change is reversible methyl and phosphate groups get detached from chromatin. (B) This model depicts that SATB1 molecules bind to DNA sequence and cause chromatin remodelling with loop like formation of DNA, analogous to noodles, like shape of DNA for ease of transcription.

the biological death of cancer patients. There are numerous basic questions that are unanswered to bring evidence of the storage and recording of cellular events, including chromatin remodeling; changes in chromosome pattern, such as coiling and uncoiling; and changes in its chemical structures, such as methylation, phosphorylation status, and interference by environmental clues, including temperature, climate, and weather. Therefore, exploring the basic mechanism of chromatin regulation and EHD within cancer cells may reveal queries about molecular and cellular events that help cancer cells thrive, which will allow therapeutic and translational experts to understand the mechanisms and behavioral patterns of cancer cells and may help in various therapeutic approaches.

\section{CHROMATIN REMODELING: AN ASPECT OF THE EPIGENOMIC HARD DRIVE}

There is knowledge that the condensed state of chromatin does not promote gene transcription. To achieve transcription, chromatin has been suggested to attain an accessible decondensed state. During epigenetic regulation, chromatin undergoes several modifications in its shape to allow the transcription factors to bind to DNA for transcription and the regulation of gene expression. ${ }^{11}$ There are various transcription factors and proteins that regulate this reshaping of chromatin. One of these DNA-binding proteins, special adenine and thymine-rich sequence binding protein 1, is also known as a chromatin organizer and regulates chromatin remodeling by loop formation of chromatin by changing gene expression. ${ }^{12-15}$ According to the present hypothesis of the existence of epigenetic recorder proteins, there may be another reality in addition to the well-known components of epigenetic regulators, such as readers, writers and erasers within the cellular landscape. These epigenetic readers, writers, erasers and recorders within normal and cancer cells may help to create EHD imprinting. We propose that EHD imprinting can store and record information about the frequency of changes in the chromatin state of cancer cells. Further, this idea of storing EHD imprinting is proposed to be complex due to the heterogeneous nature of the TME. Therefore, there is a need to understand EHD imprinting within tumor heterogeneity.

A perspective suggests that cancer cells could survive even after the biological death of cancer patients. Thus, scientific findings indicate that different types of cells are supposed to die at different time points of death events in cancer patients. Hence, 


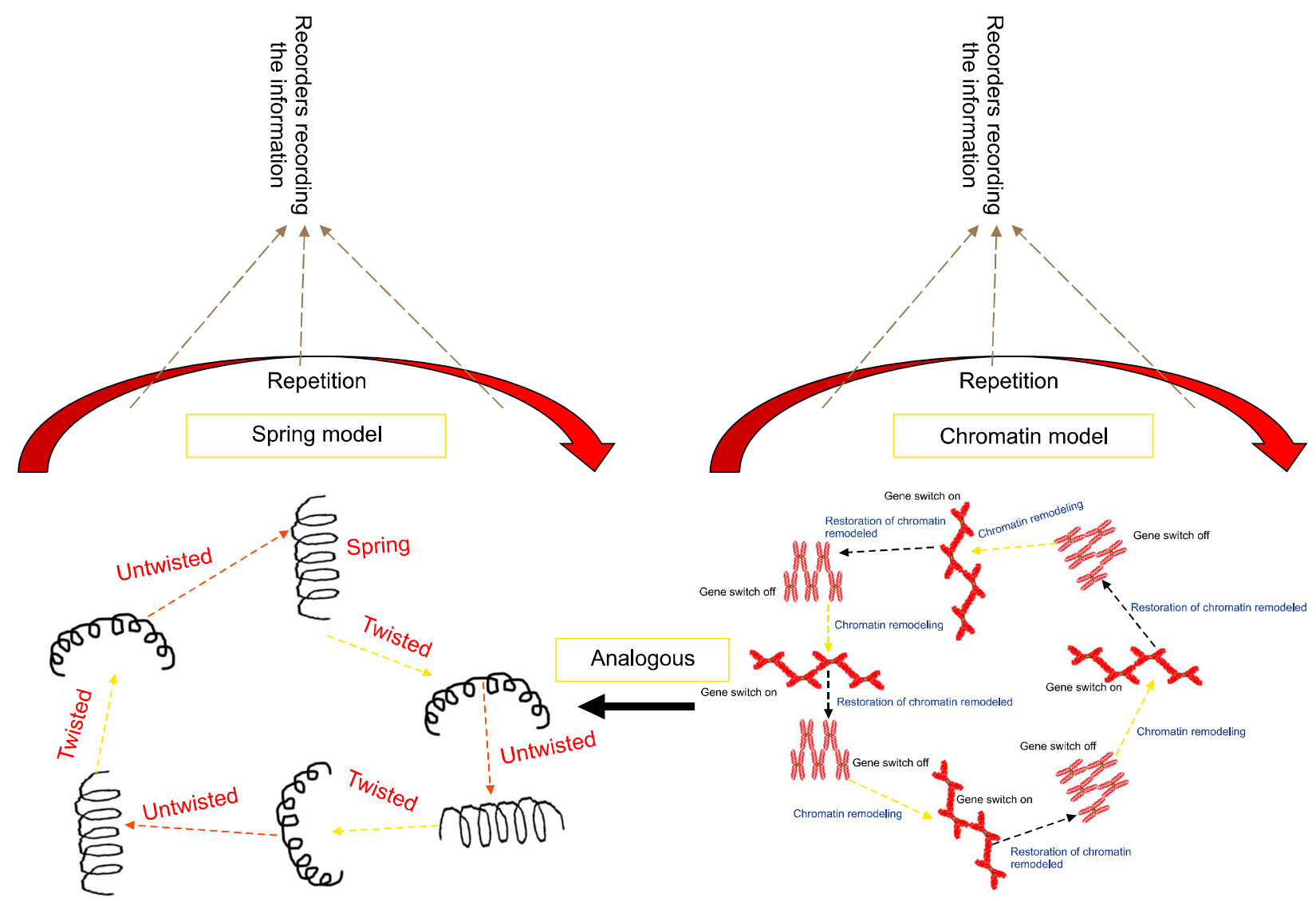

Figure 3. The analogous shape modelling/remodelling between spring and chromatin structure. Here with sequential twist and untwist of spring, texture and appearance may change, despite normal shape. Hence, there should be some recorder to monitor about the how many times this spring has gone through twist and untwist. We hypothesize that this spring model may be analogous to chromatin modelling in a cyclic and sequential manner involving writer, reader and eraser to achieve differential state of chromatin and gene expression in response to external clues. To record or store the data related to these sequential chromatin modeling, we suggest about the existence of additional mechanisms recording with a set of players able to record these chromatin alterations events.

what would be the chronological events for cancer cells during death, either early or at end of the death of all normal cells of a cancer patient. At the same, the idea should not be confused with the ability of biopsy tissues or cellular materials to grow indefinitely in an in vitro cell culture environment that does not fully replicate in vivo settings. If experimentally it may be proven that the in vivo and in vitro communication tendencies of these cancerous cells differ from normal and obedient cellular community members, then a pertinent question is to reveal the nature of signaling molecules in cancer cells, even after the demolition of cellular organization within a body. The role of nutrient storage in EHD imprinting may enable cancer cells to survive after the biological death of cancer patients. We suggest exploring the synchronous or asynchronous nature of cancer cell death and any additional survival strategies beyond the biological death of cancer patients. An interesting possibility about cancer cells is that these cells may have the ability to connect with outside clues to maintain the immortal nature. Therefore, we suggest the use of experimental tools and techniques to study the biopsy tissue samples of cancer patients to reveal the diverse array of communicating and survival clues in the form of EHD imprinting, including non-coding regulatory RNAs, chromatin landscape, intracellular signaling networks, and extracellular signals. An unanswered query may also be resolved to show the survival duration of cancer cells after the biological death of cancer patients and the link with EHD imprinting. In addition to the fate of cancer cells, a small fraction of tumor cells, as cancer stem cells, could also be investigated for their contributions in cellular events during the biological death of cancer patients. With all of these queries of basic understanding and translational interest, we propose exploring EHD imprinting. 


\section{CONTRIBUTION OF CELLULAR MICROENVIRONMENT TO EPIGENOMIC HARD DRIVE IMPRINTING}

Cancer cells live in a microenvironment that comprises a heterogeneous nature creating TME. There are various enzymes, proteins, peptides and small RNAs to shape TME that may contribute to epigenetic modifications. Among several types of epigenetic regulatory pathways, histone post-translational modification, induction of hypomethylation or hypermethylation, phosphorylation, acetylation of genes, such as DNA (cytosine-5)methyltransferase 3-like, promyelocytic leukemia-retinoic acid receptor alpha fusion protein, trithorax group proteins and polycomb group protein have been reported in various cancer types. ${ }^{16-19}$ These epigenetic modifications either cause transcriptional repression or gene activation by methylation, histone protein modifications, chromosome remodeling and other epigenetic alterations. These forms of cellular heterogeneity have been suggested to be driven by autocrine and paracrine signaling pathways. Importantly, these distinct signaling pathways play a role in the modulation of chromatin status and such changes can be stored in the form of EHD imprinting.

\section{STATE OF TUMOR EPIGENETIC HETEROGENEITY BEFORE AND AFTER BIOLOGICAL DEATH}

After the biological death of cancer patients, heterogeneous cancer cells could be deprived of extracellular nutrition, such as glucose, essential amino acids, and cofactors including ATP, $\mathrm{NADPH}$, Acetyl-CoA, and $\mathrm{NAD}^{+} .{ }^{20}$ Other than nutrition, chemical and physical signals from the external environment are also missing, which is crucial for cell proliferation and survival. In the condition of death, both normal and cancer cells are supposed to undergo a change in their survival mechanisms mediated by signaling, genetic and epigenetic pathways. In essence, the tendency of cancer cells to be immortal can be linked to a distinction of EHD imprinting over normal cells.

\section{UNDERSTANDING EPIGENOMIC HARD DRIVE DYNAMICS BY NON-CELLULAR FACTORS}

Changes in the chromosome pattern are also caused by non-cellular factors encompassing various stimuli, such as signals produced by the surroundings or other cells and adaptations of receptors in response to environmental factors, including temperature, climate, weather, stress and physiological disorders and nutritional habits. ${ }^{16,21-26}$ In addition to these physical and chemical factors, psychological factors, such as happiness, anxiety, stress, depression, and social burden, can result in chromatin shape and structural changes, as well as chemical modifications of chromatin such as methylation and phosphorylation. ${ }^{27}$ Those genetic changes may be recorded in the chromatin structure as an EHD by epigenetic recorder cellular proteins. In addition, the regulation of EHD imprinting can be impacted by natural compounds as active substances derived from natural sources, such as plants, fungi, and marine life forms, which modulate and highlight the importance of EHD modulation in the perspective of cancer therapeutics. ${ }^{28,29}$

\section{UNDERSTANDING THE EPIGENOMIC HARD DRIVE USING AN IN VITRO 3-DIMENSIONAL TUMOR MODEL}

The TME comprises the extracellular matrix (ECM), blood vasculature, and supporting stromal cells. The physical characteristics of the ECM, cellular components and secreted non-cellular factors have been proposed to contribute towards epigenetic changes. In turn, these microenvironmental factors have been indicated to play a vital role in controlling cancer cell proliferation, apoptosis, metabolism and differentiation. ${ }^{30} \mathrm{~A}$ vast array of materials and fabrication techniques have been employed to develop scaffolds with varying physical and biological characteristics to address cellular architecture of the human body. Among various available methods, naturally derived ECM-based hydrogels are most commonly used for in vitro applications of 3-dimensional (D) cell culture. Tumor cells typically form spheroids when grown in 3-D suspension cultures or within hydrogels. Cells within the spheroids are heterogeneous and organize in a manner that enables gradient formation and diffusion dynamics that correspond to in vivo settings within tumors. The inner core of the spheroids exhibits a hollow lumen resembling the necrotic areas of in vivo cancers. ${ }^{31}$ These areas are hypoxic and are usually observed at a distance from nutrient and oxygen supplies. In addition, the proliferation of tumor cells cultured in 3-D is typically slower than that observed in monolayer cultures.

To understand the EHD imprinting of tumors, there is a need to utilize a 3-D tumor culture model to closely mimic the TME. In short, the experiment could be proposed to test the deprivation of growth factors, nutrients and oxygen to match the in vivo 
clinical patient cancer model. By revealing these observations, we suggest that EHD imprinting of cancer cells would show a differential landscape of chromatin. In addition, these studies may assist in understanding the heterogeneous and complex nature of tumors and may open an avenue of precise and personalized cancer therapeutics.

\section{EXPERIMENTAL STRATEGY TO TEST EPIGENOMIC HARD DRIVE IMPRINTING IN A CANCER PATIENT MODEL}

To validate the hypothesized model to understand changes in the EHD imprinting of carcinoma tissue before and after the biological death of carcinoma patients, we suggest investigating tumor tissue samples before and after the biological death of cancer patients. Investigating their genetic and epigenetic landscapes will surely shed light on their behavioral pattern before and after biological death, i.e., what was the status of the cancer cells before death and what was the status of the cells after death.

To support the proposed hypothesis, we suggest an idea of live cell imaging with fluorescent proteins before and after the biological death of cancer patients. Live cell imaging is a technique used for studying the biological functions, genetics, and epigenetic and molecular status of cells inside the living body. ${ }^{32}$ By performing live cell imaging of cancer cells before and after the biological death of cancer patients, data will reveal EHD imprinting before and after biological death. These epigenetic changes may be recorded by recorder molecules available in the vicinity of the chromatin complex.

Further, we suggest an experimental approach in a cancer patient model by providing various stimuli emanating from the external environment in the form of, for example, nutritional factors. The effects of these factors are suggested to be studied through a live cell imaging system on the behavioral pattern and to observe the cancer cell response to these stimuli. Possible efforts may be made to check whether there are any effects of these factors on these cells, proving a role of non-cellular factors in EHD imprinting regulation. The EHD imprinted information could be retrieved using molecular-based experiments by DNA methylation, histone modifications and non-coding regulatory RNAs.

\section{CLINICAL SIGNIFICANCE OF EXPLORING THE EPIGENOMIC HARD DRIVE}

The translational values of revealing EHD imprinting could lie in understanding a unique set of small molecules secreted by tumor cells in the case of distinct pathophysiological conditions, including virus-infected cancer patients. Further, a proposed study on EHD imprinting will allow for an understanding of the leaching out potentials of these deleterious agents to the environment, and in turn, this study will help in the management of health and diseases. Other significant knowledge of EHD imprinting may help to characterize defensive and survival molecular agents employed by cancer cells. In the future, this knowledge can be translated to conceive new pharmacological inhibitors against various cancer types. In addition to the translation value, mining the tumor tissues before and after biological death may provide extraordinary clues about the tumorigenic potentials, specifically in the setting of EHD information. Another scope is proposed to determine the cellular and non-cellular factors used to achieve the distinct state of EHD imprinting in cancer patients. Further, we propose that the evaluation of EHD imprinting may help to decide various modalities including nutrition, social environment, stress-free surroundings and other potential factors that can extend the life of cancer patients.

Further, EHD imprinting will also shed light on intricacies about the network of signaling pathways and an array of stimuli exchanged reversibly between the environment and TME. The knowledge about EHD imprinting will help to identify and target these survival pathways employed by cancer to exist after the biological death of patients. Thus, EHD imprinting may serve as a clue for the development of various therapeutic approaches being taken to dismantle the robust and aberrant cellular signaling cascades within cancer cells and the TME. New therapeutic approaches for the treatment of cancer can be harnessed as a new class of drugs or inhibitors as an importantly epigenetic imprinting decoder or retriever. Based on the above basic and translational potential, exploring EHD imprinting in cancer could open new approaches and interests in cancer research. In addition, the idea of EHD imprinting could be extrapolated to other human disease models to uncover basic facts about unanswered cellular complexities.

\section{CONCLUSION}

In conclusion, we hypothesize that $\mathrm{EHD}$ imprinting may reveal crucial questions to understand tumor heterogeneity and its implications before and after the biological death of cancer patients. We propose that cancer cells, being part of a human cellular community, may carry some encrypted coded message in 
the form EHD imprinting. Further, we also speculate about the existence of epigenomic recorders, in line with known and existing molecules, such as epigenetic writers, readers and erasers, to modulate the epigenetic status. This new concept of EHD imprinting and epigenetic recorders could be helpful to reveal cellular and physiological events that occur before and after the biological death of cancer patients. We suggest that epigenetic tools, such as the assessment of DNA methylation, histone code signature, and small regulatory miRNAs, could be performed upon cancerous and non-cancerous tissues grouped before and after the biological death of cancer patients. New cutting-edge tools and techniques may decipher the epigenetic landscape that may provide answers to the above-proposed concept of EHD imprinting and epigenetic recorders. These revelations and insights could help pave the way to a better understanding of cancer cells, the biological death process and a futuristic new class of therapeutic inhibitors.

\section{ACKNOWLEDGMENTS}

The authors acknowledge financial support from DST-SERB, Government of India, New Delhi, India (SERB/LS-1028/2013) and Dr. D. Y Patil, Vidyapeeth, Pune, India (DPU/05/01/2016).

\section{CONFLICTS OF INTEREST}

No potential conflicts of interest were disclosed.

\section{REFERENCES}

1. Pattabiraman DR, Weinberg RA. Tackling the cancer stem cells: what challenges do they pose? Nat Rev Drug Discov 2014;13: 497-512.

2. Fuchs $\mathrm{Y}$, Steller H. Live to die another way: modes of programmed cell death and the signals emanating from dying cells. Nat Rev Mol Cell Biol 2015;16:329-44.

3. Feinberg AP, Koldobskiy MA, Göndör A. Epigenetic modulators, modifiers and mediators in cancer aetiology and progression. Nat Rev Genet 2016;17:284-99.

4. Moran S, Martínez-Cardús A, Sayols S, Musulén E, Balañá C, Estival-Gonzalez A, et al. Epigenetic profiling to classify cancer of unknown primary: a multicentre, retrospective analysis. Lancet Oncol 2016;17:1386-95.

5. Torres CM, Biran A, Burney MJ, Patel H, Henser-Brownhill T, Cohen AS, et al. The linker histone H1.0 generates epigenetic and functional intratumor heterogeneity. Science 2016;353: aaf1644.

6. Jones PA, Baylin SB. The epigenomics of cancer. Cell 2007;128: 683-92.

7. Allis $\mathrm{CD}$, Jenuwein $\mathrm{T}$. The molecular hallmarks of epigenetic control. Nat Rev Genet 2016;17:487-500.
8. Van Tongelen A, Loriot A, De Smet C. Oncogenic roles of DNA hypomethylation through the activation of cancer-germline genes. Cancer Lett 2017:396:130-7.

9. Erlich Y, Zielinski D. DNA Fountain enables a robust and efficient storage architecture. Science 2017;355:950-4.

10. De Silva PY, Ganegoda GU. New trends of digital data storage in DNA. Biomed Res Int 2016;2016:8072463.

11. Geiman TM, Robertson KD. Chromatin remodeling, histone modifications, and DNA methylation-how does it all fit together? J Cell Biochem 2002;87:117-25.

12. Yasui D, Miyano M, Cai S, Varga-Weisz P, Kohwi-Shigematsu T. SATB1 targets chromatin remodelling to regulate genes over long distances. Nature 2002;419:641-5.

13. Cai S, Lee CC, Kohwi-Shigematsu T. SATB1 packages densely looped, transcriptionally active chromatin for coordinated expression of cytokine genes. Nat Genet 2006;38:1278-88.

14. Lee JJ, Kim M, Kim HP. Epigenetic regulation of long noncoding RNA UCA1 by SATB1 in breast cancer. BMB Rep 2016;49:578-83.

15. Hamm CA, Stevens JW, Xie H, Vanin EF, Morcuende JA, Abdulkawy $\mathrm{H}$, et al. Microenvironment alters epigenetic and gene expression profiles in Swarm rat chondrosarcoma tumors. BMC Cancer 2010;10:471.

16. Manderwad GP, Gokul G, Kannabiran C, Honavar SG, Khosla S, Vemuganti GK. Hypomethylation of the DNMT3L promoter in ocular surface squamous neoplasia. Arch Pathol Lab Med 2010; 134:1193-6.

17. Subramanyam D, Belair CD, Barry-Holson KQ, Lin H, Kogan SC, Passegué E, et al. PML-RAR\{alpha\} and Dnmt3al cooperate in vivo to promote acute promyelocytic leukemia. Cancer Res 2010;70:8792-801.

18. Richly H, Aloia L, Di Croce L. Roles of the Polycomb group proteins in stem cells and cancer. Cell Death Dis 2011;2:e204.

19. Sparmann A, van Lohuizen M. Polycomb silencers control cell fate, development and cancer. Nat Rev Cancer 2006;6:846-56.

20. Keibler MA, Wasylenko TM, Kelleher JK, Iliopoulos O, Vander Heiden MG, Stephanopoulos G. Metabolic requirements for cancer cell proliferation. Cancer Metab 2016;4:16.

21. Stein RA. Epigenetics and environmental exposures. J Epidemiol Community Health 2012;66:8-13.

22. Feil R, Fraga MF. Epigenetics and the environment: emerging patterns and implications. Nat Rev Genet 2012;13:97-109.

23. Hou L, Zhang X, Wang D, Baccarelli A. Environmental chemical exposures and human epigenetics. Int J Epidemiol 2012;41: 79-105.

24. Toraño EG, García MG, Fernández-Morera JL, Niño-García P, Fernández AF. The impact of external factors on the epigenome: in utero and over lifetime. Biomed Res Int 2016:2016:2568635.

25. Romani M, Pistillo MP, Banelli B. Environmental epigenetics: crossroad between public health, lifestyle, and cancer prevention. Biomed Res Int 2015:2015:587983.

26. Herceg Z. Epigenetic mechanisms as an interface between the environment and genome. Adv Exp Med Biol 2016;903:3-15.

27. Zannas AS, Chrousos GP. Epigenetic programming by stress and glucocorticoids along the human lifespan. Mol Psychiatry 2017; 22:640-46.

28. Ratovitski EA. Anticancer natural compounds as epigenetic modulators of gene expression. Curr Genomics 2017;18:175-205.

29. Hahn O, Grönke S, Stubbs TM, Ficz G, Hendrich O, Krueger F, et al. Dietary restriction protects from age-associated DNA methyl- 
ation and induces epigenetic reprogramming of lipid metabolism. Genome Biol 2017;18:56.

30. Xu X, Farach-Carson MC, Jia X. Three-dimensional in vitro tumor models for cancer research and drug evaluation. Biotechnol Adv 2014:32:1256-68
31. Yamada KM, Cukierman E. Modeling tissue morphogenesis and cancer in 3D. Cell 2007;130:601-10.

32. Hoffman RM. Live cell imaging in live animals with fluorescent proteins. Methods Enzymol 2012;506:197-224. 